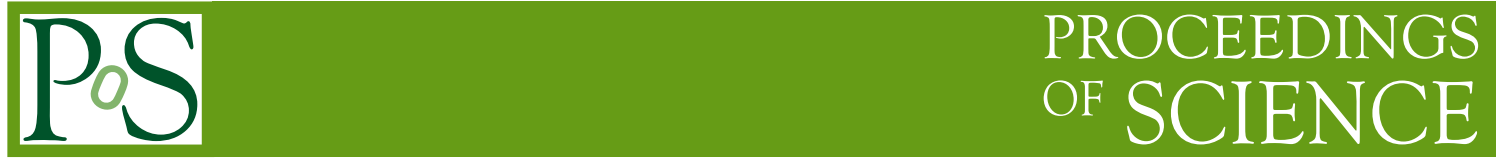

\title{
Matching the lattice coupling to the continuum for the tree level Symanzik improved gauge action
}

\author{
Michele Brambilla* \\ University of Parma and INFN \\ E-mail: michele.brambilladfis.unipr.it \\ Francesco Di Renzo \\ University of Parma and INFN \\ E-mail: francesco.direnzodfis.unipr.it
}

We present current results for the matching between the $\overline{M S}$ scheme and the lattice scheme definied by the tree-level Symanzik improved gauge action and $n_{f}=2$ Wilson fermions.

We exploit the knowledge of the matching between $\alpha_{\overline{M S}}$ and $\alpha_{V}$ and numerically compute the matching between $\alpha_{V}$ and $\alpha_{T L S}$.

The XXVIII International Symposium on Lattice Field Theory, Lattice2010

June 14-19, 2010

Villasimius, Italy

\footnotetext{
${ }^{*}$ Speaker.
} 


\section{Introduction}

In order to extract physical information from lattice simulation the relationship between the bare lattice and the renormalized coupling is an essential ingredient. One is in particular interested in the $\overline{M S}$ coupling, the most widely used in particle physics. Its matching to the lattice regularization defined by the tree level Symanzik gauge action and Wilson fermions is known up to one loop (see for example [1]). Can a higher order knowledge of the relationship be useful? The answer is yes: the computation is not only interesting on its own, but also a requisite for matching observables or renormalization coefficients at high loop.

We are indeed interested in computing the three loop renormalization coefficients $Z_{\Gamma}$ in the RI'-MOM scheme [2] ( $\Gamma$ stands for a generic current). This can be done since the anomalous dimensions are known up to three loops [ $[$ ] [ [丹]: in force of this, it is possible to fix the logarithms in $Z_{\Gamma}$. Since anomalous dimensions are known as expansions in a continuum $\alpha_{s}$, one needs a (two loop) matching between the lattice and continuum coupling constants.

The general form of the matching between two schemes is given by

$$
\alpha(s \mu)=\alpha^{\prime}(\mu)+c_{1}(s) \alpha^{\prime}(\mu)^{2}+c_{2}(s) \alpha^{\prime}(\mu)^{3}+\ldots,
$$

where $s$ is a coefficient that accounts for the choice of a different scale, and the coefficients $c_{1}(s)$ and $c_{2}(s)$ are given by

$$
\begin{aligned}
& c_{1}(s)=2 b_{0} \log \frac{\Lambda}{\Lambda^{\prime}}-2 b_{0} \log s \\
& c_{2}(s)=c_{1}(s)^{2}-2 b_{1} \log s+2 b_{1} \log \frac{\Lambda}{\Lambda^{\prime}}+\frac{b_{2}-b_{2}^{\prime}}{b_{0}} .
\end{aligned}
$$

$b_{0}, b_{1}$ and $b_{2}$ are coefficients of the $\beta$ function, and $\Lambda$ is the scale associated to the regularization. It is worth to remember that $b_{0}$ and $b_{1}$ are universal, while $\Lambda$ and $b_{2}$ depend on the scheme. Eq. (1.3) states that the two loop matching of $\alpha_{T L S}$ to $\alpha_{\overline{M S}}$ also entails the knowledge of $b_{2}^{T L S}$, since $b_{2}^{\overline{M S}}$ is known. This is the purpose of this work.

\section{Method}

One could of course envisage a direct matching of $\overline{M S}$ to the tree level Symanzik scheme. We will go through a different strategy, matching first to an intermediate scheme. As it will be clear, we will need no computation of logarithms. Since we make use of Numerical Stochastic Perturbation Theory (NSPT) [5], this would require a terrific numerical precision.

Following [6] we compute the static quark potential from Wilson loops and Creutz ratio:

$$
V_{T}(R)=\log \left(\frac{W(R, T-1)}{W(T, R)}\right)
$$

which in turn can yield the static potential via

$$
V(R)=\lim _{T \rightarrow \infty} V_{T}(R)
$$


The static quark potential is the quantity which describes the interaction energy of a infinitely heavy $q \bar{q}$ pair. In its full (non-perturbative) form, it is in first approximation just the sum of a string tension, which is responsible for confinement, and a $R^{-1}$ contribution, whose interpretation is different in different IR/UV regimes

$$
V(R)=\frac{C}{R}+\sigma R
$$

In the perturbative (UV) regime the first term is just the Coulomb potential, but using the definitions (2.1) and (2.2) one is left in addition with a linearly divergent term, which gives the so called residual mass of the heavy quark:

$$
V(R)=2 \delta m-C_{F} \frac{\alpha_{V}(R)}{R} .
$$

Eq. (2.4) defines the potential coupling $\alpha_{V}(R)$ we will be concerned with.

A perturbative computation of the static quark potential in our lattice scheme reads

$$
V(R)=2 \delta m-C_{F} \frac{\alpha_{T L S}}{R}\left(1+c_{1}(R) \alpha_{T L S}+c_{2}(R) \alpha_{T L S}^{2}+\mathscr{O}\left(\alpha_{T L S}^{3}\right)\right),
$$

and comparing with (2.4) one can read the matching (1.1) of the renormalized $\alpha_{V}(R)$ in terms of (the bare) $\alpha_{T L S}$

$$
\alpha_{V}(R)=\alpha_{T L S}+c_{1}(R) \alpha_{T L S}^{2}+c_{2}(R) \alpha_{T L S}^{3}+\mathscr{O}\left(\alpha_{T L S}^{4}\right) .
$$

In this case (1.2) and (1.3) read

$$
\begin{aligned}
& c_{1}(R)=2 b_{0} \log \frac{\Lambda_{V}}{\Lambda_{T L S}}+2 b_{0} \log R \\
& c_{2}(R)=c_{1}(R)^{2}+2 b_{1} \log R+2 b_{1} \log \frac{\Lambda_{V}}{\Lambda_{T L S}}+\frac{b_{2}^{(V)}-b_{2}^{(T L S)}}{b_{0}} .
\end{aligned}
$$

As a byproduct of the computation we also obtain the residual mass $\delta m$ as an expansion in $\alpha_{T L S}$

$$
\sum_{n \geq 0} \delta m^{(n)} \alpha_{T L S}^{n+1}
$$

Once we have computed the matching between $\alpha_{V}(R)$ and $\alpha_{T L S}$, we need the matching of $\alpha_{V}(R)$ to $\alpha_{\overline{M S}}$. This can be read from the computation in [7].

\section{Computation}

By making use of NSPT, we computed the Wilson loops $W(R, T)$ for all the values of $R$ and $T$ up to 16 on a $32^{4}$ lattice. The quark mass was set to zero by plugging the appropriate mass counterterm for the perturbative critical mass [8]. Results were averaged over $\sim 150$ lattice configurations.

We extracted both the residual quark mass and the coefficient we are interested in by fitting (order-by-order) our data to the expected form defined by eq. (2.5).

Given the finite lattice size nature of our computation we can not actually take neither the $T \rightarrow \infty$ limit nor the continuum limit. In other words, we expect that results are distorted by lattice artifacts. In order to minimize these effects we consider an interval of $R$ such that 
- $T>R(T / R \sim 2.5)$;

- $R$ itself is not too small $(R \geq 3)$;

- the fitting intervals themselves are from 3 up to 7 points long.

Results are of course affected by both systematic (lattice artifacts) and statistical errors. The relative weigth of these effects is different for different orders. This actually opens the way to looking for a careful tradeoff between the errors.

We adopt the following strategy: when systematic effects are clearly distinguishable (i.e. statistical effects are relatively small), we only consider $T=16$ data. This is the case of the tree level potential (figure 1): the different $V_{T}(R)$ are separated curves (though they intersect within errors).

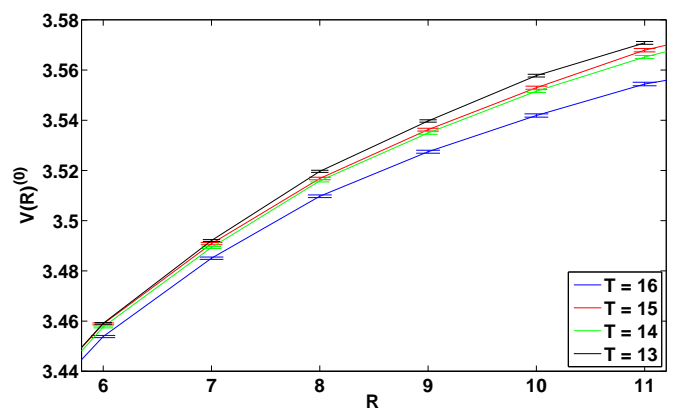

Figure 1: Tree level potential. The different lines represent the $V_{T}(R)$ extracted form different values of $T$, from $T=13$ (higher) to $T=16$ (lower).

When statistical errors are significant on their own, we need a different approach. In figure 2 we show the two loop potential. $V_{T}(R)$ for different values of $T$ widely intersect within errors; in other terms, the systematic (finite $T$ ) effect is not that clear. In this case we decided to neglect this systematic effect and tame the statistical noise by averaging over different values of $T$ (starting from $T=14$ to $T=16$ ). In other words, we thus obtain smoother curves.

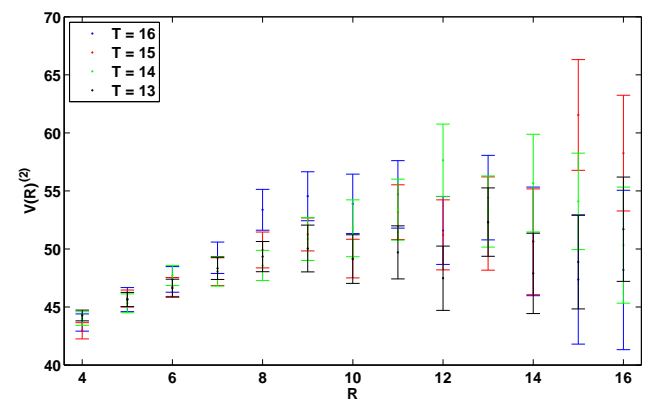

Figure 2: Two loop potential. In this case statistical errors are higher than systematic one, making hard to distinguish them. 


\section{Result}

In order to verify the reliability of this computation we first checked known results. The correctness of this is not trivial, since at any order residual mass is unknown, and we get it as a byproduct. We estimate $3 \leq R \leq 7$ as the best fitting interval for tree level and one loop; the same interval was also taken for two loop computation.

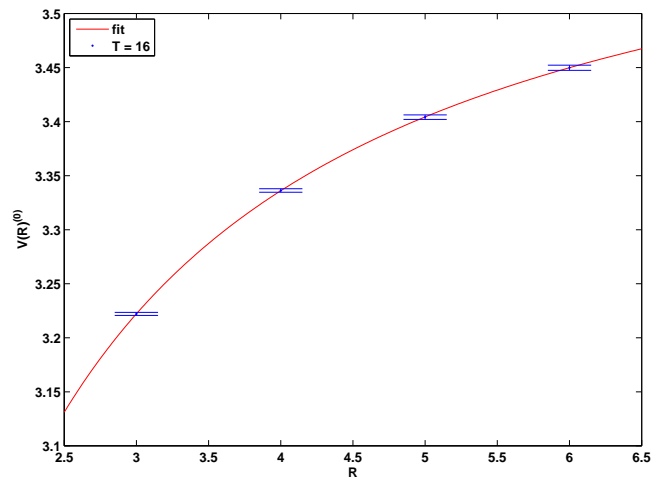

Figure 3: Data and fit (continuous line) for tree level potential.

In figure 3 we show tree level data, to be fitted to the functional form

$$
V(R)^{(0)}=2 \delta m^{(0)}-\frac{C_{F}}{R} .
$$

We obtain $\delta m^{(0)}=1.84 \pm 0.01$, while $C_{F}$ is reconstructed to a few percent. This gives a rough idea of the impact of systematic effects.

In figure 7 we show one loop data.

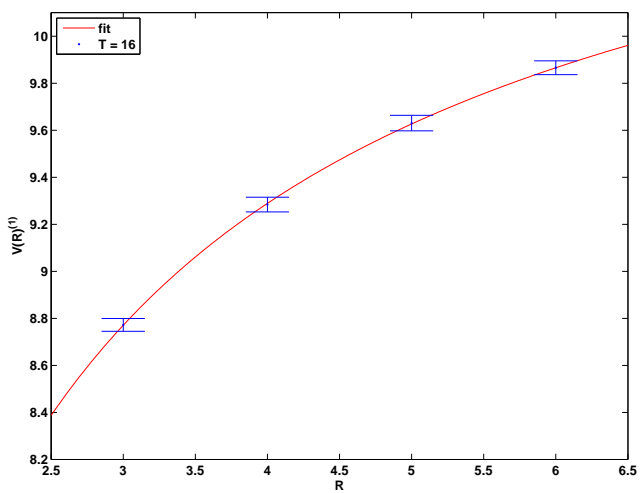

Figure 4: One loop potential: data and fit.

At one loop we are able to extract the constant term $\log \frac{\Lambda_{V}}{\Lambda_{(T L S)}}$, which we can compare to the analytical result:

$$
V(R)^{(1)}=\delta m^{(1)}-\frac{C_{F}}{R} 2 b_{0}\left(\log R+\log \frac{\Lambda_{V}}{\Lambda_{T L S}}\right) .
$$


We obtain $\log \frac{\Lambda_{V}}{\Lambda_{(T L S)}}=2.8 \pm 0.1$, to be compared to the analytical result 2.8191 [1]. We also obtain $\delta m^{(1)}=5.71 \pm 0.01$.

At two loop we finally tackle the determination of the quantity we are interested in $\left(\frac{b_{2}^{(V)}-b_{2}^{(T L S)}}{b_{0}}\right)$ :

$$
V(R)^{(2)}=\delta m^{(2)}-\frac{C_{F}}{R}\left(c_{1}(R)^{2}+2 b_{1} \log R+2 b_{1} \log \frac{\Lambda_{V}}{\Lambda_{T L S}}+\frac{b_{2}^{(V)}-b_{2}^{(T L S)}}{b_{0}}\right) .
$$

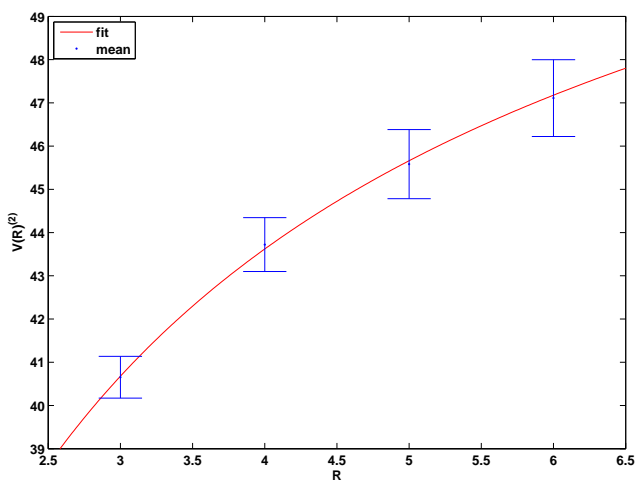

Figure 5: Two loop potential: data and fit.

In figure 5 one can see that two loop fluctuations are larger than at lower orders, and as a consequence the fit will suffer of a larger indetermination. In this case we obtain $\delta m^{(2)}=30 \pm 1$ and

$$
\frac{b_{2}^{(V)}-b_{2}^{(T L S)}}{b_{0}}=4 \pm 1 .
$$

Though the relative error in this value is high, we must emphasize that we are interested in the whole coefficient of the $\alpha^{3}$ term, in particular in the final matching to $\alpha_{\overline{M S}}$

$$
\alpha_{\overline{M S}}=\alpha_{T L S}+2.79866 \alpha_{T L S}^{2}+11.5( \pm 1.0) \alpha_{T L S}^{3}+\mathscr{O}\left(\alpha_{T L S}^{4}\right)
$$

where the relative error on the second coefficient is less than $10 \%$. While we are trying to increase our accuracy, one should keep in mind that what we are really interested in is the impact of this matching in the logarithmic contribution to the $Z_{\Gamma}$ we mentioned at the very beginning.

\section{Acknowledgements}

This work is supported by I.N.F.N. under the research project MI11 and by the Research Executive Agency (REA) of the European Union under Grant Agreement number PITN-GA-2009238353 (ITN STRONGnet). 


\section{References}

[1] S. Aoki, K. i. Nagai, Y. Taniguchi and A. Ukawa, Perturbative renormalization factors of bilinear quark operators for improved gluon and quark actions in lattice QCD, Phys. Rev. D 58 (1998) 074505 [arXiv:hep-lat/9802034].

[2] F. Di Renzo, M. Brambilla and L. Scorzato, Perturbative vs non-perturbative renormalization: the case of the quark mass PoS LAT2010 225.

[3] J. A. Gracey, Three loop anomalous dimension of non-singlet quark currents in the RI' scheme, Nucl. Phys. B 662 (2003) 247 [arXiv:hep-ph/0304113].

[4] K. G. Chetyrkin and A. Retey, Renormalization and running of quark mass and field in the regularization invariant and MS-bar schemes at three and four loops, Nucl. Phys. B 583 (2000) 3 [arXiv:hep-ph/9910332].

[5] F. Di Renzo and L. Scorzato, Numerical stochastic perturbation theory for full QCD, JHEP 0410 (2004) 073 [arXiv:hep-lat/0410010].

[6] F. Di Renzo, L. Scorzato, The Residual mass in lattice heavy quark effective theory to alpha**3 order, JHEP 0102 (2001) 020. [hep-lat/0012011]. F. Di Renzo, L. Scorzato, The $N(f)=2$ residual mass in perturbative lattice-HQET for an improved determination of $m(b) * *$ anti-MS $(m(b) * *$ anti-MS), JHEP 0411 (2004) 036. [hep-lat/0408015].

[7] Y. Schroder, The two-loop static potential, Nucl. Phys. Proc. Suppl. 86 (2000) 525 [arXiv:hep-ph/9909520].

[8] A. Skouroupathis, M. Constantinou, H. Panagopoulos, Two-loop additive mass renormalization with clover fermions and Symanzik improved gluons, Phys. Rev. D77 (2008) 014513 [arXiv:0801.3146 [hep-lat]]. 